Check for updates

Cite this: Chem. Sci., 2018, 9, 7779

๑ All publication charges for this article have been paid for by the Royal Society of Chemistry

Received 30th July 2018

Accepted 21st August 2018

DOI: $10.1039 / c 8 s c 03382 j$

rsc.li/chemical-science

\section{High yield accelerated reactions in nonvolatile microthin films: chemical derivatization for analysis of single-cell intracellular fluid + t}

\author{
Zhenwei Wei, ${ }^{a}$ Xiaochao Zhang, ${ }^{\mathrm{b}}$ Jinyu Wang, ${ }^{\mathrm{b}}$ Sichun Zhang, (DD ${ }^{\mathrm{b}}$ Xinrong Zhang ${ }^{\star \mathrm{b}}$ \\ and R. Graham Cooks (D) *a
}

The identification of trace components from biological media can require derivatization under mild conditions for successful analysis by mass spectrometry (MS). When aqueous droplets (ca. $500 \mathrm{~nL}$ ) containing a sugar and an amine as reagents are allowed to evaporate they may form long-lasting microthin films in which derivatization reactions can occur fast relative to reaction rates in bulk solution. Evidence is presented that these reactions are heterogeneous in nature and comparisons are made with reactions in pastes and in neat reagent mixtures. Moreover, these thin film reactors can be made stable for the long periods of time that may be necessary to give high product yields. The situation is typified by imine formation from reducing sugars which have reaction times of much more than 1 hour provided that small concentrations (e.g. 20 ppm) of nonvolatile solvents are included. After evaporation of almost all the water, the reaction occurs at an approximately constant rate for the first hour. The rate is two orders of magnitude faster than the reaction in the corresponding homogeneous saturated bulk solution. Conversion of the reagent to the Schiff base product is $67 \%$ to $96 \%$ efficient in these long-lasting thin films, in sharp contrast to the corresponding derivatization efficiencies in the bulk of less than $1 \%$. This method was used to chemically derivatize and thus to identify, using tandem mass spectrometry, 29 reducing sugars in ca. $1 \mathrm{~nL}$ of intracellular fluid from a single onion epidermis cell. A formal description of the kinetics of reversible and irreversible second order reactions in thin films is provided. The effects of thermodynamic and kinetic factors are separated and the measured apparent acceleration factor is shown to represent the ratio of intrinsic rate constants for the microthin film reactor relative to the bulk reaction.

\section{Introduction}

Chemical derivatization is an important strategy to increase analytical performance, ${ }^{\mathbf{1 - 5}}$ especially analytical sensitivity and selectivity, ${ }^{6,7}$ and it has been widely used for profiling metabolites, ${ }^{\mathbf{8} 9}$ quantifying bioactive small molecules and identifying natural products. ${ }^{\mathbf{1 0 - 1 2}}$ Recently, new demands have been placed on chemical derivatization in the analysis of small amounts of compounds in microvolume samples derived from individual cells. ${ }^{13}$ Especially significant on very small scales is the fact that derivatization can lead to loss of analytes due to unsatisfactory reaction yields. The problems in utilizing chemical

${ }^{a}$ Department of Chemistry, Purdue University, West Lafayette, IN 47907, USA. E-mail: cooks@purdue.edu

${ }^{b}$ Department of Chemistry, Tsinghua University, Beijing Key Lab Microanalytical Methods and Instruments, Beijing 100084, P. R. China. E-mail: xrzhang@tsinghua. edu.cn

$\dagger$ Dedicated to Keith R. Jennings, a pioneer in mass spectrometry, on his $85^{\text {th }}$ birthday.

\$ Electronic supplementary information (ESI) available. See DOI: $10.1039 / \mathrm{c} 8 \mathrm{sc} 03382 \mathrm{j}$ derivatization for small volume assays, especially in single-cell analysis, can be alleviated by addressing two questions: (1) can derivatization reactions be accelerated? (2) Can the yields of derivatization reactions be improved?

In the recent past, several novel routes to access fast reactions and unique reactivity have been reported. For example, in "on water" chemistry, organic reactions take place in an aqueous/organic emulsion. These reactions exhibit reaction rate acceleration and unique reactivity compared to bulk reactions conducted in solvents or under neat conditions. ${ }^{\mathbf{1 4 , 1 5}}$ Very recently, prebiotic mimic chemistry has established that phosphorylation of nucleosides can proceed rapidly and in high yield in an aqueous paste of highly concentrated reactants with minimum solvent. ${ }^{16}$ In these two cases, reaction acceleration and unique reactivity take place in a solvent-poor system where high concentrations and limited solvation drive the reaction. Another somewhat similar case uses flow chemistry in an ionic liquid droplet reactor to catalyze reactions and achieve higher rates and conversion ratios with a special reactive surface. ${ }^{17,18}$ Compared to homogeneous bulk phase reactions, these emerging studies using highly reactive interfaces lead to high 
reaction rates and favorable product yields. In addition to these cases, reactions are also known to be accelerated by one to five orders of magnitude ${ }^{19}$ relative to those in bulk when they take place in small confined volumes such as in electrosprayed microdroplets, ${ }^{20-25}$ levitated neutral droplets ${ }^{26-28}$ or thin films..$^{\mathbf{2 0 2 8 - 3 0}}$ In each of these systems, the reaction mixture undergoes solvent evaporation to generate large surface to volume ratio compartments where reactants are concentrated and rate constants may be increased by interfacial effects. ${ }^{31}$

When manipulating single cell samples for chemical analysis dilution is usually involved and this decreases the already low concentrations of target molecules. Pre-concentration of compounds derived from single cells may be necessary to achieve satisfactory derivatization yields. Reactions in microdroplets or thin films are especially attractive for such cases because solvent evaporation will not only bring reactant concentration into a useful range but the increased surface-tovolume ratio will also increase rate constants and reaction yields. As accelerated reaction rates and satisfactory yields follow upon solvent evaporation, the issue is how to effectively control solvent evaporation to make the system stable in the solvent-poor state so as to drive the reaction at a high rate and achieve satisfactory yields. In the past few years, efforts have been made by our groups and others ${ }^{26-30,32,33}$ to achieve this goal. For example, in Leidenfrost droplet synthesis, the solvent can be added back continuously to the levitated droplet to compensate for the solvent loss by evaporation, thus increasing the reaction time in a small controlled-volume reactor. ${ }^{26}$ In thin film deposition synthesis, ${ }^{29}$ small amounts of the reaction mixture are continually added to create a 'living' thin film of the precipitated product on an inert surface, again increasing the reaction time for continuous synthesis while retaining the small volume feature needed for acceleration.

Here, we introduce a reaction strategy for chemical derivatization in ultra-small volumes using a nonvolatile microthin film reactor. Reaction in the microthin film is accelerated while the presence of traces of a nonvolatile solvent in the microthin film increases the reaction time to help achieve very high derivatization yields. The solvent-poor nature of the film means that the equilibrium conditions do not lead to a significant reverse reaction. To characterize the performance of such a microthin film reactor, the Schiff base reaction between a set of primary amines and saccharides (Scheme 1) was studied and the data are compared to bulk reactions run under traditional bulk, neat, and paste conditions. The reasons for this particular choice are (i) Schiff base formation is a reversible reaction with a modest equilibrium constant $\mathrm{t}^{\mathbf{3 4 , 3 5}}$ and a reaction rate constant which makes it very difficult to perform chemical derivatization in small volume samples, (ii) identification and quantification of bioactive molecules like saccharides in tissues and single cells is an important task which could lead to deeper understanding of metabolic pathways, ${ }^{\mathbf{8}, 9}$ cell heterogeneity ${ }^{\mathbf{3 6}-40}$ and even to the discovery of new drugs ${ }^{10-12}$ and (iii) imine formation is an attractive route to efficient ionization of sugars.

\section{Results and discussion}

\section{Performing reactions in microthin film reactors}

Microthin film reactors were created by allowing evaporation of almost all the water from a $500 \mathrm{~nL}$ droplet of an aqueous reaction mixture containing $100 \mu \mathrm{M}$ nonvolatile amine (ca. 20 ppm, $10 \mathrm{pL}$ ) and $100 \mu \mathrm{M}$ saccharide at $25^{\circ} \mathrm{C}$ (Fig. 1). Note that very little reaction occurs while most of the $500 \mathrm{~nL}$ droplet evaporates. Then, because the aliphatic amines are both nonvolatile and polar, they keep the solvent from evaporating completely so allowing the reaction to proceed in the resulting microthin film reactor for an hour or more. After a chosen reaction time, the reaction is quenched by adding $500 \mathrm{~nL}$ water. The quenched solution is subsequently analyzed by nanoelectrospray mass spectrometry (nESI-MS) in the variant that uses an inductive DC voltage. ${ }^{\mathbf{4 1}}$ The apparent acceleration factor (AAF) is used to evaluate reactions in different systems. It is measured as the ratio of ratios of ion abundances of the product $[\mathrm{P}]$ relative to the starting material $[\mathrm{SM}]$, viz. $([\mathrm{P}] /[\mathrm{SM}])$ in the thin film relative to that in bulk after the same reaction time: ${ }^{\mathbf{2 8 , 2 9}}$

$$
\mathrm{AAF}=\frac{\left(\frac{[\mathrm{P}]}{[\mathrm{SM}]}\right)_{\text {thin film }}}{\left(\frac{[\mathrm{P}]}{[\mathrm{SM}]}\right)_{\text {bulk }}}
$$

The usefulness of this system for efficient chemical derivatization and chemical analysis is shown below by the highly sensitive mass spectrometric analysis of reducing saccharides in a single Allium cepa cell.

\section{Comparison of microthin film reactions to bulk reactions under different conditions}

Fig. 2 shows kinetic data (basis spline fitted) for the reaction between glucose and $N, N$-dibutyl-1,3-propanediamine (DBPA) at

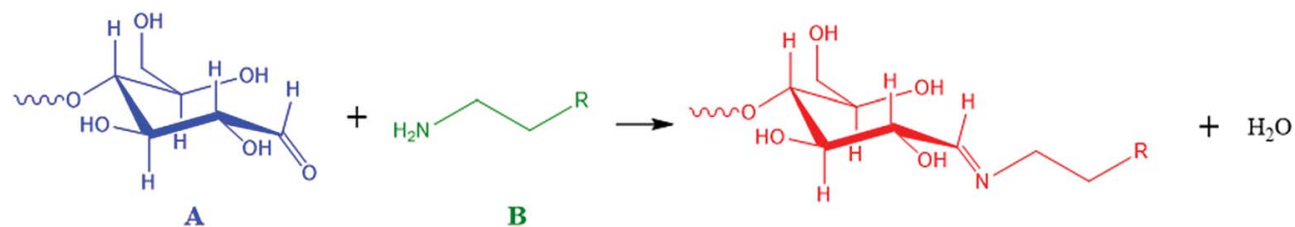


- $500 \mathrm{~nL}$ water droplet

- $100 \mu \mathrm{M}$ reactants

- 20 ppm nonvolatile solvent

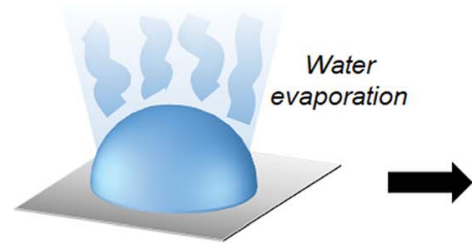

Microthin film formation
- Microthin film reactor

- Concentrated reactants

- Ca. $10 \mathrm{pL}$ nonvolatile solvent

- Very less amount water

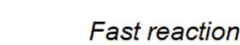

Unique reactivity

Stable for an hour

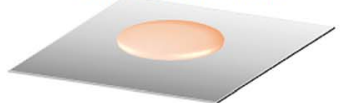

Microthin film Reactor

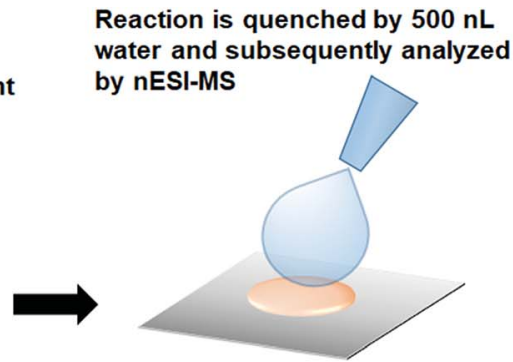

Product characterization

Fig. 1 Construction of a microthin film reactor for fast reaction and long reaction times. Typically, a $500 \mathrm{~nL}$ aqueous reaction mixture (100 $\mu \mathrm{M}$ saccharide and amine) was dropped on a plate covered with parafilm and left to dry. For amines with high vapor pressure, 20 ppm DMF was added to increase the reaction time. No pH adjustment was made. Accelerated reaction rates and increased extents of reaction could be achieved in the microthin film reactor for an hour. The reaction is allowed to proceed in this reactor for times ranging from several minutes to several hours and subsequently analyzed by $\mathrm{nESI}-\mathrm{MS}$. Before MS measurement, the sample from the microthin film was diluted to $500 \mathrm{~nL}$ to quench the reaction.

room temperature $\left(25^{\circ} \mathrm{C}\right)$ from 0 min to $70 \mathrm{~min}$. These mass spectrometric measurements (detailed in ESI, Fig. S1 supplemented by optical microscopy. Note that this reaction was run in the microthin film for up to 24 hours but only data for the first hour have been chosen to examine the reaction kinetics because the microthin thin film dimensions did not change perceptibly during this period. Schiff base reactions are usually second order $^{34}$ so the ratio of product ion intensity/ starting material ion intensity, viz., ([P]/[SM]), measured from the corresponding ion signals in MS, is plotted against the reaction time to give the kinetics curve. (See ESI, $\$$ for additional discussion, including the derivation of the equation showing the linear dependence of $[\mathrm{P}] /[\mathrm{SM}]$ on both the rate constant and the initial concentration.) The kinetics curves of the reaction in the microthin film show two zones. Zone 1 starts with the deposition of the $500 \mathrm{~nL}$ droplet and lasts about 7 minutes, while almost all the water evaporates. Microscopy (Fig. S2\$) showed that the reaction system was still in the form of a droplet of a large cross section and MS showed that the extent of reaction was very low during this stage. Zone 2 begins at about 7 minutes, which is when the reaction system was observed to change from a droplet to a microthin film (Fig. S2 From the microscopy image in Fig. S2, the cross-sectional shape of the thin film in a typical experiment was constant from $8 \mathrm{~min}$ to $70 \mathrm{~min}$. The thin film thickness is not known but it is many thousands of monolayers even after an hour. The kinetics curves in zone 2 are linear, indicating that the reaction occurred under kinetic control over a period of at least $60 \mathrm{~min}$ (from 7 $\min$ to $70 \mathrm{~min}$ ). The kinetics curve in zone 2 is used to compare with the kinetics curves of bulk reactions in saturated solution and neat reactants and under "paste" (solvent-poor) conditions. The AAF can be measured from the ratio of the slopes of the kinetics plots in zone $2 v s$. that of bulk reactions recorded under these different conditions, provided that the system is under kinetic control (see the detailed discussion of the kinetic control region in the ESI:):

$$
\mathrm{AAF}=\frac{\left(\frac{[\mathrm{P}]}{[\mathrm{SM}]}\right)_{\text {thin film }}}{\left(\frac{[\mathrm{P}]}{[\mathrm{SM}]}\right)_{\text {bulk }}}=\frac{k_{\mathrm{f}} c_{0, \mathrm{f}}}{k_{\mathrm{b}} c_{0, \mathrm{~b}}}
$$

where $k_{\mathrm{f}}$ and $k_{\mathrm{b}}$ are the thin film and bulk rate constants and $c_{0, \mathrm{f}}$ and $c_{0, \mathrm{~b}}$ are the initial concentrations in film and bulk media (see the ESIt for derivation and more detailed discussion). From Fig. 1b, among these four reaction conditions, the microthin film reaction appears to have the fastest reaction rate (slope $=7.48 \times 10^{-2}, R^{2}=0.9799$, and $\mathrm{RSD}=5.6 \%$ ), which is $3.0(\mathrm{RSD}=5.6 \%)$ times faster than the paste reaction (slope $=2.52 \times 10^{-2}, R^{2}=0.9903$, and RSD $\left.=3.6 \%\right), 14.2$ times ( $\mathrm{RSD}=3.6 \%)$ faster than the neat reaction (slope $=5.24$ $\times 10^{-3}, R^{2}=0.9718$, and $\left.\mathrm{RSD}=3.2 \%\right)$ and 230 times ( $\mathrm{RSD}=$ $14.5 \%$ ) faster than the bulk reaction at $25 \mathrm{mM}$ with saturated DBPA (slope $=3.25 \times 10^{-4}, R^{2}=0.9747$, and RSD $=14.5 \%$ ). As the solubility of DBPA in water is not good, $25 \mathrm{mM}$ is the maximum concentration possible for the homogenous bulk reaction. The significant difference in the reaction rate between the microthin film reaction and the bulk reaction with saturated DBPA suggests a heterogeneous reaction mechanism of the fast reaction in the microthin film. It is notable that the heterogeneous reaction mechanism is also used to describe the reaction under neat and paste conditions. ${ }^{14,15}$ In contrast to the neat and paste reactions, in the microthin film reaction the reaction mixture is derived from solvent evaporation of a homogeneous droplet, ensuring that reactants in the heterogeneous microthin film are well mixed. Evaporation enhances mass transfer efficiency through preconcentration. This accounts for an increase in the reaction rate in the microthin film reactor by a factor of 2 over the paste reaction and almost 10 over the neat reaction. According to eqn (2), the AAF in different media is determined by the ratio of the intrinsic reaction rate constants and by the initial reagent concentration. In the microthin film, neat and paste reactions, the reactants are highly concentrated and oversaturated, and therefore the ratio of concentrations is $c a .1$ and under these circumstances, the AAF actually reflects the difference in intrinsic rate constants.

\section{Solvent effect on microthin film reactions}

Further information on the role of solvation in reactions in microthin films comes from experiments using solvents of different vapor pressures and polarities to construct the 
a) Reaction Acceleration When Micro Thin Film Formed

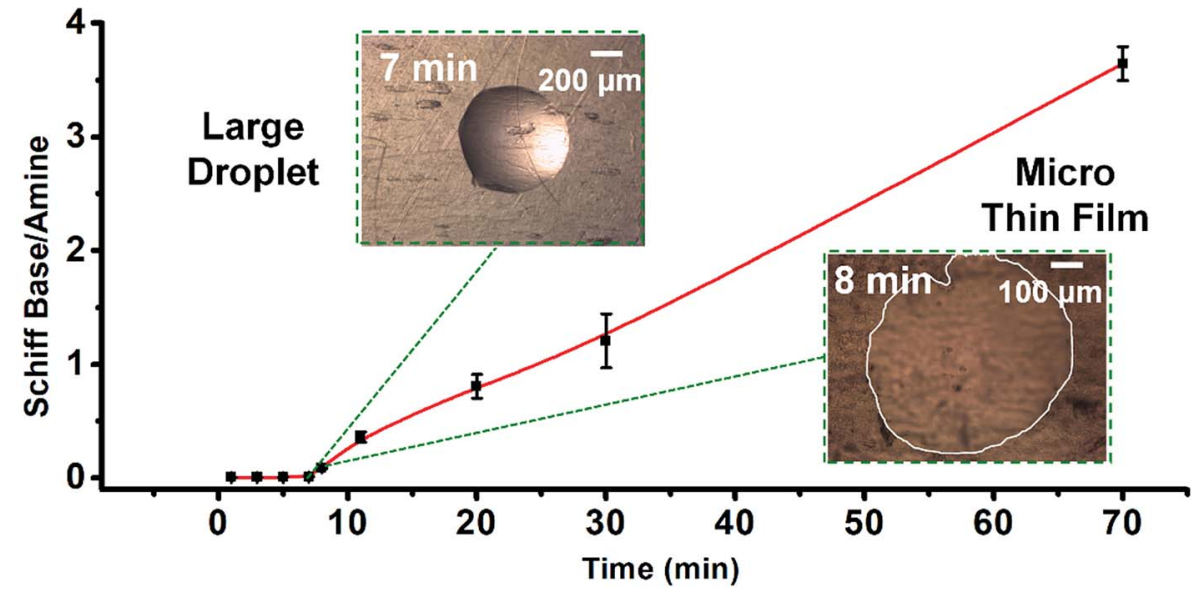

b)

25 mM Bulk Reaction

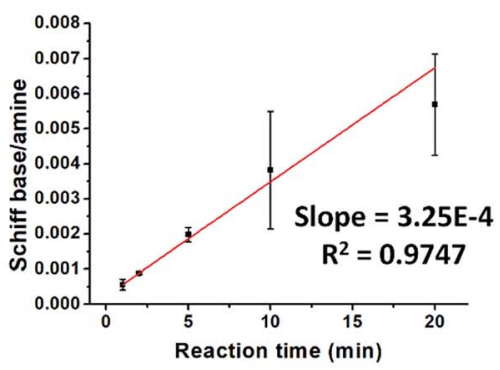

Paste Reaction

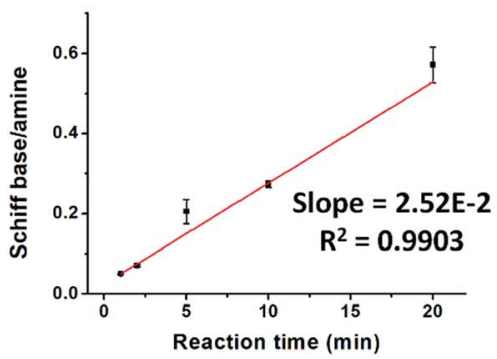

Neat Reaction
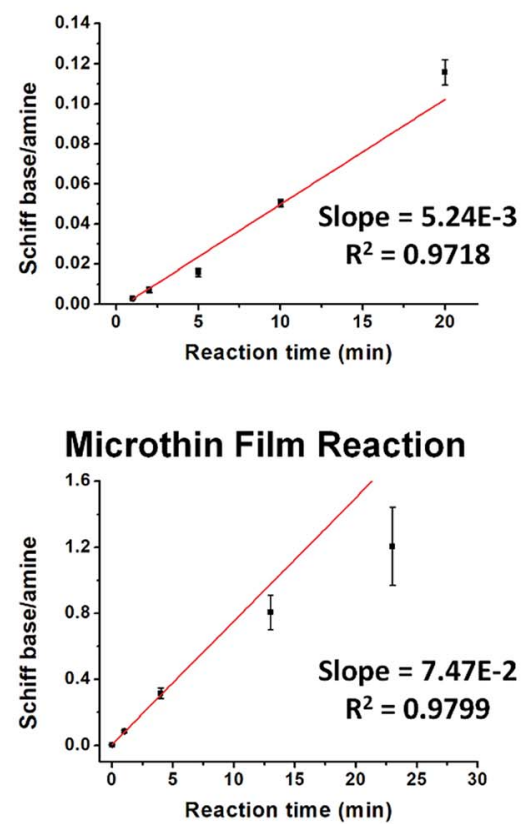

Fig. 2 (a) Kinetics of Schiff base reaction in a $500 \mathrm{~nL}$ microdroplet under ambient conditions, showing product Schiff base intensity relative to reactant amine intensity. (b) Kinetics curves (fitted based on a second-order rate equation) of bulk reactions between glucose and DBPA under different conditions: saturated bulk reaction (25 mM DBPA and $25 \mathrm{mM}$ glucose), neat reaction (1 mmol glucose and $1 \mathrm{mmol}$ DBPA mixed in a 1.5 $\mathrm{mL}$ Eppendorf tube and stirred), paste reaction ( $1 \mathrm{mmol}$ glucose, $1 \mathrm{mmol}$ DBPA and $100 \mu \mathrm{L}$ water mixed in a $1.5 \mathrm{~mL}$ Eppendorf tube and stirred) and microthin film reaction $(100 \mu \mathrm{M}$ glucose and $100 \mu \mathrm{M}$ DBPA mixture in a $500 \mathrm{~nL}$ droplet and left in an incubator to dry to form a microthin film reactor). Note that the kinetics curves of all bulk reactions fall into the kinetic control region. Since the downward deflection of kinetics curves in the microthin film reactions is due to thermodynamic considerations (see the detailed discussion of the kinetic control region in the ESI the first four points in the microthin film reaction are fitted. All data were obtained from three replicate experiments.

microthin film. In Table 1, using nonvolatile reagents, we found that solvents of higher vapor pressure facilitate microthin film reactions, probably because of the increased concentration when less solvent remains in the microthin film reactor at $25^{\circ} \mathrm{C}$. In detail, water, isopropanol and methanol, with increasing vapor pressures of 23.7 torr, 45.4 torr and 127 torr, show increasing conversion ratios after a 20 min reaction of $55 \%$, $78 \%$ and $87 \%$, respectively. An extreme case is that of DMF, a nonvolatile solvent, which showed a conversion ratio of less than $1 \%$. Besides vapor pressure, polar solvents show significantly higher conversion ratios than nonpolar solvents. For example, although cyclohexane and hexane have standard vapor pressures of 96.9 torr and 153 torr, their conversion ratios are only $5 \%$ and $9 \%$. This is consistent with more effective solvation of the amino group of DBPA and the hydroxyl groups of glucose by the polar solvent, making it easier for the amino group to attack glucose. 
Table 1 Conversion ratio $([\mathrm{P}] /([\mathrm{SM}]+[\mathrm{P}]))$ for reactions between DBPA and glucose using different solvents ${ }^{a}$

\begin{tabular}{llll}
\hline Solvent name & Polarity & $\begin{array}{l}25{ }^{\circ} \mathrm{C} \text { vapor } \\
\text { pressure (torr) }\end{array}$ & $\begin{array}{l}\text { Conversion } \\
\text { ratio (\%) }\end{array}$ \\
\hline Methanol & Polar & 127 & $86 \pm 2$ \\
Isopropanol & Polar & 45.4 & $75 \pm 4$ \\
Water & Polar & 23.7 & $51.2 \pm 4$ \\
DMF & Polar & 3.87 & $<0.1$ \\
CHEX & Nonpolar & 96.9 & $6 \pm 3$ \\
Hex & Nonpolar & 153 & $9 \pm 1$
\end{tabular}

${ }^{a}$ Total reaction time was $20 \mathrm{~min}$ ( $8 \mathrm{~min}$ for droplet evaporation and 12 min in the microthin film form); conversion ratio was reported here to emphasize yield differences between different systems. Data were obtained from three replicate experiments.

\section{Temperature effects on microthin film reactions}

The temperature was also examined as a variable affecting thin film reactivity. When the reaction between DBPA and glucose was performed in a microthin film at $65^{\circ} \mathrm{C}$, the kinetics curve (Fig. 3a) again showed very good linearity between the reaction time and ratio, $[\mathrm{P}] /[\mathrm{SM}]$. However, the slope was $1.23 \mathrm{~min}^{-1}\left(R^{2}=0.9973\right.$, $\mathrm{RSD}=3.1 \%$ ), which is 24.4 times that for the reaction at $25^{\circ} \mathrm{C}$. A common explanation of the accelerated reaction rate is the increase of the intrinsic rate constant at the higher reaction temperature. However, we also found that less solvent is left in the microthin film reactor at a higher temperature (Fig. S4 $\ddagger$ ) and suggest that this also contributes to the observed rate increase. The advantage of increased temperature is clear but at about 60 minutes rupture of the thin film was observed so that it no longer had a single continuous surface. The reaction between glucose and amines of different vapor pressures at $65^{\circ} \mathrm{C}$ showed values of $[\mathrm{P}] /[\mathrm{SM}]$ after $20 \mathrm{~min}$ of reaction that correlated negatively with the amine vapor pressure (Fig. S5 ). Several other observations suggested that the nonvolatile primary amine plays a key role in stabilizing the microthin film. Use of large vapor pressure amines or decreasing the amine amount made the thin film extremely fragile. This problem could be solved by using mixed solvents to

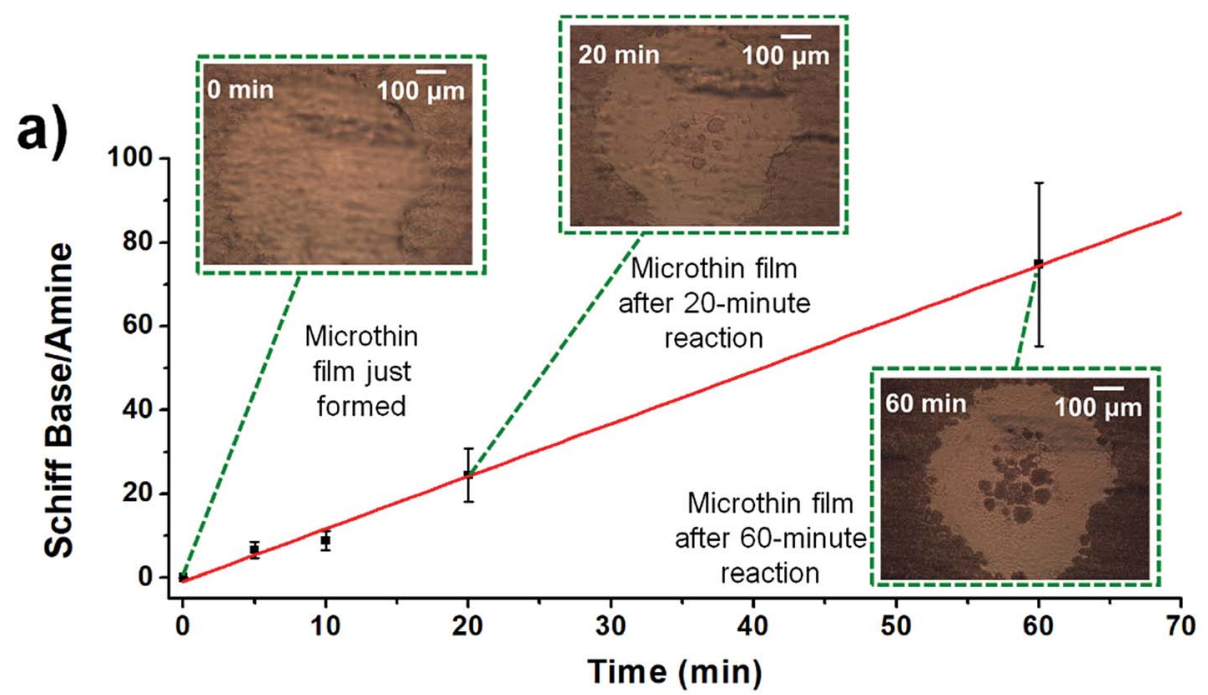

b)
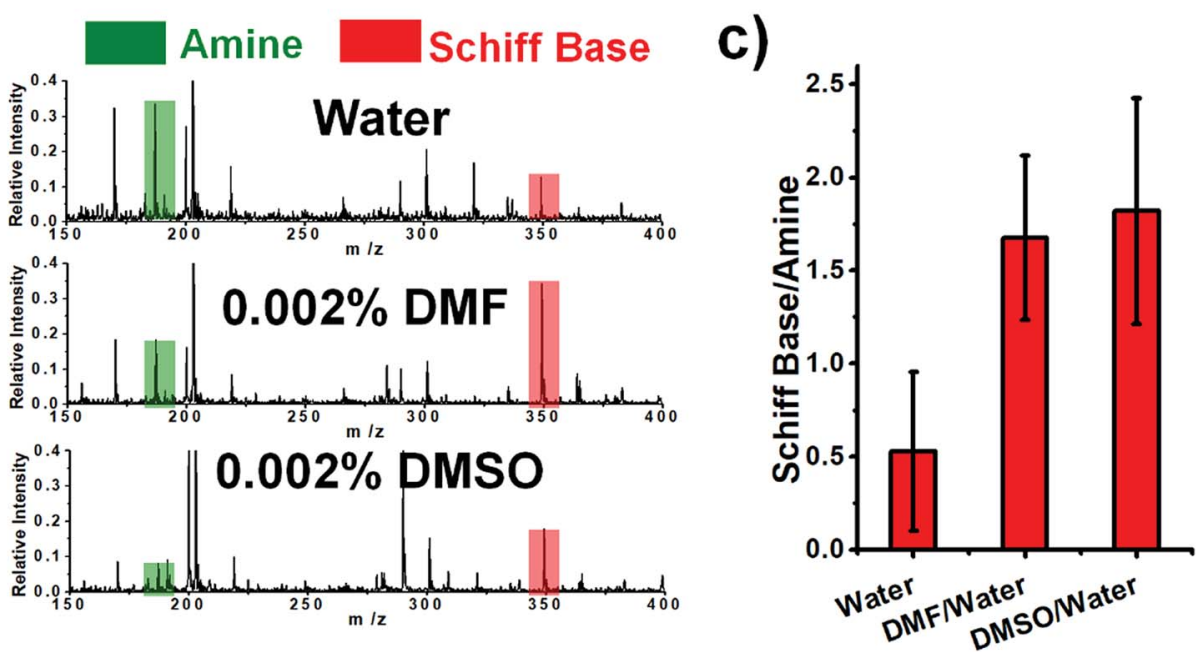

Fig. 3 (a) Kinetics curve for the reaction between aqueous DBPA and glucose in $65^{\circ} \mathrm{C}$. The droplet was first held at $25^{\circ} \mathrm{C}$ for 8 min to ensure that the microthin film had formed and then held at $65^{\circ} \mathrm{C}$ for the reaction. (b) Schiff base reaction between $100 \mu \mathrm{M}$ glucose and $10 \mu \mathrm{M}$ DBPA in the microthin film for $10 \mathrm{~min}$ with water, $0.002 \%$ DMF in water and $0.002 \%$ DMSO in water as the solvent. (c) [P]/[SM] after a 10 min reaction in different solvents. All data were obtained from three replicate experiments. 
generate the thin film. When using just $0.002 \%(\mathrm{v} / \mathrm{v})$ DMF or DMSO in the DBPA/glucose reaction, $[\mathrm{P}] /[\mathrm{SM}]$ increased from 0.5 (water) to 1.7 and 1.8, respectively (Fig. $3 \mathrm{~b}$ and c). Besides amine vapor pressure, amine basicity may also influence the reaction. For the nonvolatile amines DMEA, DEEA, DEPA and DBPA, $\mathrm{p}_{\mathrm{a}_{1}}$ is 6.6, 7.0, 7.7 and 8.0 (data source: https:/www.chemicalize.com), respectively, which correlates positively with the $[\mathrm{P}] /[\mathrm{SM}]$ value of 9.0, 15.9, 18.7 and 21.3. However, basicity is a secondary factor relative to vapor pressure. For example, although butylamine, a volatile amine, has a $\mathrm{p} K_{\mathrm{a}}$ of 10.2 the $[\mathrm{P}] /[\mathrm{SM}]$ value is only 0.34 .

\section{Reaction yields in microthin film reactions}

Besides reaction acceleration, an even more important characteristic of microthin film reactors, which could have broad

Table 2 Conversion ratio (CR) for reactions between DBPA and different saccharides in a traditional bulk reactor and a microthin film reactor. Both reactions were at $65^{\circ} \mathrm{C}$ and data were taken at 24 hours for the bulk reaction and 20 minutes for the microthin film reaction

\begin{tabular}{lll}
\hline Saccharides & $\begin{array}{l}\text { CR (\%) in } \\
\text { bulk }(24 \text { hours })\end{array}$ & $\begin{array}{l}\text { CR (\%) in microthin } \\
\text { films (20 minutes) }\end{array}$ \\
\hline Glucose & $0.5 \pm 0.3$ & $95.7 \pm 0.4$ \\
Maltose & $0.5 \pm 0.2$ & $90.2 \pm 1.2$ \\
Maltotriose & $0.4 \pm 0.2$ & $86.3 \pm 1$ \\
Maltotetraose & $0.5 \pm 0.2$ & $72.3 \pm 2.6$ \\
Maltopentaose & $0.4 \pm 0.2$ & $70.4 \pm 5.8$ \\
Maltohexose & $0.4 \pm 0.1$ & $64.9 \pm 10.1$
\end{tabular}

application, is the significant yield enhancement available. High yields depend on being able to run reactions for appropriately long times, which we have seen is readily achieved in microthin film reactors. They also require large equilibrium constants, especially when the substrate is present in low concentration. The Schiff base reaction is normally not suitable for chemical derivatization because it is a reversible reaction with a small equilibrium constant under standard conditions. For example, the reaction of protonated $p$-chlorobenzaldehyde and aniline hydrochloride to yield protonated $N$-p-chlorobenzylideneaniline has an equilibrium constant of only $0.08 .^{34}$ When using $100 \mu \mathrm{M}$ DBPA to derivatize $100 \mu \mathrm{M}$ of a saccharide (glucose, maltose, maltotriose, maltotetraose, maltopentaose and maltohexaose) in bulk solution at $65{ }^{\circ} \mathrm{C}$, the $[\mathrm{P}] /[\mathrm{SM}]$ values of all six reactions after 24 hours were less than 0.005 (conversion ratio $<0.5 \%$ ) (Table 2). However, using the microthin film reactor for derivatization, the $[\mathrm{P}] /[\mathrm{SM}]$ value was 2.01 to 22.1 (conversion ratio $66.8 \%$ to $95.7 \%$ ), viz. 500 to 6000 times the bulk reaction value. Notably, the reaction takes only 20 minutes compared to 24 hours for completion in bulk. The increase in the reaction yield is the result of three features of the microthin film reactor strategy: (i) the concentrations of reactants can be as high as that in a saturated solution, (ii) the fraction of molecules near the surface is maximized, and (iii) the open reaction system promotes escape of water produced in the reaction. This latter factor increases the reaction conversion ratio and it also accelerates the reaction according to the rate equation of reversible reactions (see ESI eqn (9)-(15) for derivations and extended discussion $\ddagger)$.

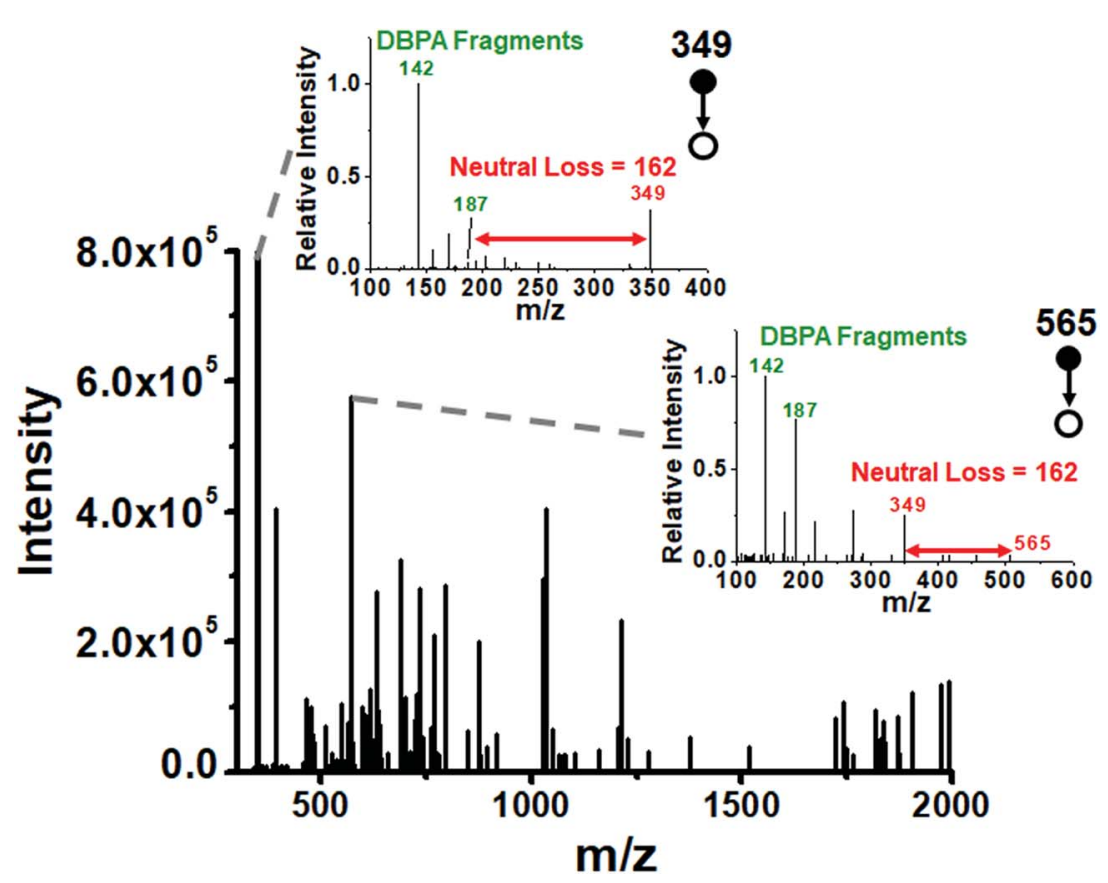

Fig. 4 Extracted $m / z$ values of two ions which undergo a neutral loss of 162 (red arrows) and DBPA derived fragments ( $m / z$ 142 and 187 ) in MS ${ }^{2}$ product ion spectra (inset) after single cell fluid derivatization. There are 125 peaks in the mass spectrum which have structures derived from DBPA and a saccharide. 


\section{Utilization of thin film reactions for derivatization of} saccharides in single cells

We utilized the microthin film reactor to perform Schiff base derivatization of the saccharides from the intracellular fluid of a single cell. The extract of an individual Allium cepa cell (ca. $1 \mathrm{~nL}$ ) was taken up in a nESI emitter tip using a 3D micromanipulator. The intracellular glucose concentrations reported for some animal cells are 2 to $50 \mathrm{mM}^{42}$ while they are $50 \mathrm{mM}$ to 125 $\mathrm{mM}^{43}$ in Allium cepa bulbs, depending on storage time. Using a value of $100 \mathrm{mM}$ as the glucose concentration, we estimate the amount of glucose in $1 \mathrm{~nL}$ of single cell extract as $18 \mathrm{ng}(0.1$ nmol) with smaller amounts of other sugars. To ensure good derivatization efficiency of all reducing saccharides in the cell, the tip of the emitter was fractured and the small sample-containing end section was immersed in a $500 \mathrm{~nL}$ droplet containing $10 \mathrm{mM}$ DBPA (50 times excess over glucose) to perform the microthin film Schiff base derivatization at $65{ }^{\circ} \mathrm{C}$ for 20 minutes (see the schematic diagram in Fig. S7 $\$$ ). The derivatized saccharides showed typical fragment ions in their product ion $\mathrm{MS}^{2}$ spectra (Fig. 4). For example, the fragment ions $m / z 142$ and 187 are characteristic imine derived fragments and the neutral loss of 162 indicates the hexose unit. We used a data dependent scan strategy to record the collision-induced dissociation of the 1050 most abundant peaks in the full scan MS; 125 of these spectra showed typical sugar neutral losses of 162 and DBPA derived fragment ions at 142 and 187. We successfully identified 29 reducing saccharides from these 125 spectra (Table S2 $\ddagger$ ), demonstrating the microthin film reactor to be a simple yet powerful tool for the rapid derivatization and identification of low nanogram level analytes in single cells.

\section{Conclusion}

The microthin film reactor utilized in this study can be contrasted with that used in an earlier study ${ }^{29}$ in which a very large acceleration factor together with continuous reagent addition was used to scale up product yields while the thin film of the reaction solution was maintained by continuous product precipitation. Product removal by precipitation there avoided concerns regarding rate decrease by the back reaction in cases where the equilibrium constant is not large. However, even if the equilibrium constant is small, as represented by this study, reaction yields can be high enough to allow successful derivatization on a picomole to nanomole scale if measures are taken to increase reaction times while working with very small amounts of reagent.

\section{Experimental section}

\section{Materials}

All saccharides (glucose, maltose, maltotriose, maltotetraose, maltopentaose and maltohexaose) were purchased from Beijing Bio-dee Biotechnology Co. Ltd. All amines (butylamine, $N, N$ dimethyl-1,2-ethylenediamine (DMEA), N,N-diethyl-1,2-diaminoethane (DEEA), $N, N$-diethyl-1,3-propanediamine (DEPA) and $N, N$-dibutyl-1,3-propanediamine (DBPA)), DMF and DMSO were purchased from Beijing Hwrk Chemical Co. Ltd. The white Allium cepa bulb was purchased from a local market. All stock solutions were stored at $4{ }^{\circ} \mathrm{C}$ before use.

\section{Reactions}

Unless otherwise noted the concentration of reactants for Schiff base reactions was $100 \mu \mathrm{M}$. For bulk reactions, a $200 \mu \mathrm{L}$ reaction mixture was placed in an Eppendorf tube in an incubator to control the reaction time and temperature. For microthin film reactions, parafilm was attached to a glass slide to form a hydrophobic surface. Then $500 \mathrm{~nL}$ of the reaction mixture was cast on the parafilm to form the reaction solution. The plate was placed in an incubator to control the temperature at either $25{ }^{\circ} \mathrm{C}$ or $65{ }^{\circ} \mathrm{C}$. For all microthin film experiments, $500 \mathrm{~nL}$ reaction solution containing all reagents is left to dry at $25^{\circ} \mathrm{C}$ for 7 minutes to form the microthin film reactors.

\section{Single cell derivatization}

A nanoelectrospray emitter was controlled by using a precise moving stage to extract $\sim 1 \mathrm{~nL}$ of fluid from a single Allium cepa cell. The end of the emitter tip was then cut off and inserted into a $500 \mathrm{~nL}$ droplet (DBPA $10 \mathrm{mM}$ ) to allow Schiff base reaction once the droplet was placed in an incubator for $10 \mathrm{~min}$ in $65^{\circ} \mathrm{C}$. This single sample was used for MS/MS based sugar and its modification identification.

\section{Mass spectrometry}

Except for the single cell analysis, all other experiments were carried out using a Thermo LTQ mass spectrometer (Thermo Scientific, San Jose CA). The instrumental parameters were as follows: capillary temperature $=275{ }^{\circ} \mathrm{C}$ and max injection time $=200 \mathrm{~ms}$. The single cell amount analysis was carried out using a Thermo QE-Orbitrap mass spectrometer (Thermo Scientific, San Jose CA). The capillary temperature was $320^{\circ} \mathrm{C}$. The analytical method was full MS, followed by data dependent $\mathrm{MS}^{2}$. The method duration was $5 \mathrm{~min}$. Full MS: resolution $=70000$, AGC $=3 \times 10^{6}$, max injection time $=$ $100 \mathrm{~ms}$, and scan range $=134$ to 2000 . The ddMS ${ }^{2}$ settings: resolution $=35000$, AGC $=1 \times 10^{5}$, ion injection time $=$ $100 \mathrm{~ms}$, loop count $=10, \mathrm{NCE}=30$, underfill ratio $=0.1 \%$, and dynamic exclusion $=300 \mathrm{~s}$.

\section{Microscopy}

A metallurgical microscope (YX20L20, Dayueweijia Science and Technology Co. Ltd., Beijing) was used to examine the microthin film. Photos of the microthin film were taken under the following conditions: reactant concentrations were $10 \mathrm{mM}$; droplet volume was $500 \mathrm{~nL}$.

\section{Conflicts of interest}

The authors declare no conflicts of interest. 


\section{Acknowledgements}

We acknowledge financial support at Purdue University from the Department of Defense, Defense Advanced Research Projects Agency (award no. W911NF-16-2-0020) and at Tsinghua University from the National Natural Science Foundation of China (award No. 21390410 and 21727813).

\section{References}

1 M. Eggink, M. Wijtmans, R. Ekkebus, et al., Anal. Chem., 2008, 80, 9042-9051.

2 D. J. Harvey, J. Chromatogr. B: Anal. Technol. Biomed. Life Sci., 2011, 879, 1196-1225.

3 J. G. Streeter and C. E. Strimbu, Anal. Biochem., 1998, 259, 253-257.

4 B. A. Bidlingmeyer, S. A. Cohen and T. L. Tarvin, J. Chromatogr., 1984, 336, 93-104.

5 A. S. A. Keyon, R. M. Guijt, C. J. Bolch, et al., Anal. Chem., 2014, 86, 11811-11818.

6 K. R. Anumula and S. T. Dhume, Glycobiology, 1998, 8, 685694.

7 S. M. Gao, Z. P. Zhang and H. T. Karnes, J. Chromatogr. B: Anal. Technol. Biomed. Life Sci., 2005, 825, 98-110.

8 S. S. Rubakhin, E. J. Lanni and J. V. Sweedler, Curr. Opin. Biotechnol., 2013, 24, 95-104.

9 B. N. Walker, J. A. Stolee and A. Vertes, Anal. Chem., 2012, 84, 7756-7762.

10 J. M. Egan, A. Kaur, H. A. Raja, et al., Phytochem. Lett., 2016, 17, 219-225.

11 J. J. Kellogg, D. A. Todd, J. M. Egan, et al., J. Nat. Prod., 2016, 79, 376-386.

12 A. Petruczynik, J. Misiurek, T. Tuzimski, et al., J. AOAC Int., 2017, 100, 1652-1659.

13 H. C. Fan, G. K. Fu and S. P. Fodor, Science, 2015, 347, 1258367.

14 S. Narayan, J. Muldoon, M. G. Finn, et al., Angew. Chem., Int. Ed., 2005, 44, 3275-3279.

15 J. E. Klijn and J. Engberts, Nature, 2005, 435, 746-747.

16 C. Gibard, S. Bhowmik, M. Karki, et al., Nat. Chem., 2018, 10, 212-217.

17 M. Zhang, R. Ettelaie, T. Yan, et al., J. Am. Chem. Soc., 2017, 139, 17387-17396.

18 M. Zhang, L. Wei, H. Chen, et al., J. Am. Chem. Soc., 2016, 138, 10173-10183.

19 S. Banerjee, E. Gnanamani, X. Yan, et al., Analyst, 2017, 142, 1399-1402.

20 X. Yan, R. M. Bain and R. G. Cooks, Angew. Chem., Int. Ed., 2016, 55, 12960-12972.
21 T. Muller, A. Badu-Tawiah and R. G. Cooks, Angew. Chem., Int. Ed., 2012, 51, 11832-11835.

22 R. M. Bain, S. Sathyamoorthi and R. N. Zare, Angew. Chem., Int. Ed., 2017, 56, 15083-15087.

23 J. K. Lee, S. Banerjee, H. G. Nam, et al., Q. Rev. Biophys., 2015, 48, 437-444.

24 S. Banerjee, E. Gnanamani, X. Yan, et al., Analyst, 2017, 142, 1399-1402.

25 I. Nam, J. K. Lee, H. G. Nam, et al., Proc. Natl. Acad. Sci. U. S. A., 2017, 114, 12396-12400.

26 Y. Li, Y. Liu, H. Gao, et al., Chem.-Eur. J., 2018, 24, 73497353.

27 E. A. Crawford, C. Esen and D. A. Volmer, Anal. Chem., 2016, 88, 8396-8403.

28 R. M. Bain, C. J. Pulliam, F. Thery, et al., Angew. Chem., Int. Ed., 2016, 55, 10478-10482.

29 Z. W. Wei, M. Wleklinski, C. Ferreira, et al., Angew. Chem., Int. Ed., 2017, 56, 9386-9390.

30 A. K. Badu-Tawiah, D. I. Campbell and R. G. Cooks, J. Am. Soc. Mass Spectrom., 2012, 23, 1461-1468.

31 Y. F. Li, X. Yan and R. G. Cooks, Angew. Chem., Int. Ed., 2016, 55, 3433-3437.

32 M. Zhang, R. Ettelaie, T. Yan, et al., J. Am. Chem. Soc., 2017, 139, 17387-17396.

33 M. I. Jacobs, J. F. Davies, L. Lee, et al., Anal. Chem., 2017, 89, 12511-12519.

34 E. H. Cordes and W. P. Jencks, J. Am. Chem. Soc., 1962, 84, 832-837.

35 F. A. Adam, M. T. Elhaty and A. A. Ibrahem, J. Chin. Chem. Soc., 1984, 31, 345-349.

36 O. Guillaume-Gentil, T. Rey, P. Kiefer, et al., Anal. Chem., 2017, 89, 5017-5023.

37 J. P. Knemeyer, D. P. Herten and M. Sauer, Anal. Chem., 2003, 75, 2147-2153.

38 C. Lombard-Banek, S. A. Moody and P. Nemes, Angew. Chem., Int. Ed., 2016, 55, 2454-2458.

39 R. M. Onjiko, S. A. Moody and P. Nemes, Proc. Natl. Acad. Sci. U. S. A., 2015, 112, 6545-6550.

40 L. Zhang and A. Vertes, Angew. Chem., Int. Ed., 2018, 57, 4466-4477.

41 Z. W. Wei, X. C. Xiong, C. A. Guo, et al., Anal. Chem., 2015, 87, 11242-11248.

42 J. E. Foley, S. W. Cushman and L. B. Salans, Am. J. Physiol.: Endocrinol. Metab., 1980, 238, E180-E185.

43 L. A. Abayomi and L. A. Terry, J. Sci. Food Agric., 2009, 89, 683-687. 